\title{
Bayesian data reduction approaches in polarization imaging
}

\author{
J. Zallat, Y. Gaudeau, C. Heinrich and S. Faisan \\ LSIIT-UMR CNRS - ULP 7005, 67412, Illkirch, France
}

\begin{abstract}
The Stokes-Mueller formalism provides a coherent framework that permits obtaining polarization parameters from raw radiance acquisitions through a linear observation model. In polarization imaging, this observation model is extended to the whole image pixels where classical pixel-wise data reduction approaches are used. The whole system's optimization relies on defining well behaved "polarization measurement matrix" that reduces the impact of noises and system errors. The richness of the spatial distribution of the information is not considered this way. Furthermore, even for a well calibrated imaging polarimeter, errors and noises, inherently related to the physics of the measurement, may be amplified that prevent the physical admissibility of the desired quantities (Stokes vectors, Mueller matrices). General Bayesian approaches to estimate polarization parameters in Stokes and Mueller imaging yield a neat solution to the polarimetric data reduction problem that preserves the physical admissibility constraints and provides robust estimates of polarization channels in regard to image noises. These approaches are evaluated by using synthetic simulated data and real measurements. We show that these methods can be used as a valuable tool to analyze physical contents of observed scenes in the framework of Stokes and Mueller imaging.
\end{abstract}

\section{References}

1. J. Zallat, S. Aïnouz and M. P. Stoll, J. Opt. A: Pure Appl. Opt., 8, 807 (2006)

2. J. Zallat and C. Heinrich, Opt. Express, 15, 83 (2007)

3. J. Zallat, C. Heinrich and M. Petremand, Opt. Express, 16, 7119 (2008) 